\title{
Efecto de la biofibra de queratina en las propiedades de un biopolímero termoplástico: Estudio preliminar
}

\section{Effect of keratin biofiber on the properties of a thermoplastic biopolymer: Preliminary study}

\author{
Sánchez-Olivares Guadalupe \\ Centro de Innovación Aplicada \\ en Tecnologías Competitivas \\ Dirección de Investigación, Posgrado y Capacitación, León, Gto. \\ Correo: gsanchez@ciatec.mx

Medina-Torres Luis
Universidad Nacional Autónoma de México
Facultad de Química
Correo: luismt@unam.mx
Calderas Fausto
Centro de Innovación Aplicada
en Tecnologías Competitivas
Dirección de Investigación, Posgrado y Capacitación, León, Gto.
Correo: fcalderas@ciatec.mx

\section{Medina-Torres Luis} \\ Universidad Nacional Autónoma de México \\ Facultad de Química \\ Correo: luismt@unam.mx \\ Calderas Fausto \\ en Tecnologías Competitivas \\ Correo: fcalderas@ciatec.mx
}

\author{
Sánchez-Solis Antonio \\ Universidad Nacional Autónoma de México \\ Instituto de Investigaciones en Materiales \\ Correo: sancheza@unam.mx \\ Moreno-Morales Rigel Leonardo \\ Universidad Nacional Autónoma de México \\ Instituto de Investigaciones en Materiales \\ Correo: leonardinogdn@gmail.com

\section{Ramírez-González Víctor} \\ Centro de Innovación Aplicada \\ en Tecnologías Competitivas \\ Dirección de Investigación, Posgrado y Capacitación, León, Gto. \\ Correo: vramirez@ciatec.mx
}

\section{Manero Octavio}

Universidad Nacional Autónoma de México

Instituto de Investigaciones en Materiales

Correo:manero@unam.mx

\section{Resumen}

En el presente trabajo se evaluó el efecto de la biofibra de queratina, obtenida de los desechos de la industria de la curtiduría de México, en un biopolímero termoplástico a base de almidón. Los materiales compuestos se obtuvieron por extrusión reactiva. Se utilizaron aditivos retardantes a la flama libres de compuestos halogenados para estudiar el efecto de la biofibra de forma comparativa. Los estudios termogravimétrico y reológico revelaron que la biofibra proporciona mayor estabilidad térmica al biopolímero, particularmente en combinación con un aditivo retardante a la flama, ya que se reduce la rapidez de pérdida de masa. Esto se atribuyó a la formación de estructuras más complejas resistentes a la alta temperatura. El análisis dinámico-mecánico, mostró que la biofibra tiene un efecto importante en el módulo de almacenamiento ( $\left.E^{\prime}\right)$, ya que se incrementa considerablemente cuando la biofibra se adiciona en combinación con los aditivos retardantes a la flama. La evaluación de las propiedades mecánicas (módulo de Young, resistencia a la tensión, deformación a la ruptura y tenacidad), confirmaron el efecto de la biofibra como agente de carga. Por otro lado, cuando la biofibra se adiciona sin aditivo retardante a la flama, la deformación a la ruptura y la tenacidad se incrementan considerablemente, lo cual se atribuyó a una buena adhesión de la biofibra con la matriz polimérica.

Descriptores: biofibra, queratina, biopolímero, estabilidad térmica, refuerzo mecánico, desechos de la curtiduría.

\begin{abstract}
In this work, the effect of keratin biofiber on the properties of a thermoplastic starch biopolymer (TPS) is investigated. The keratin biofiber was obtained from tannery industry waste. The materials were compounded by reactive extrusion and injection molding. Traditional halogen-free Flame Retardant (FR) additives were used for comparison. According to the thermogravimetric and rheological analyses, the addition of the keratin biofiber increases the thermal stability of the TPS, especially in combination with FR additives, since the mass loss rate was reduced. This behavior is attributed to the formation of more complex and resistant structures in the composite material. From dynamic-mechanical analysis, the keratin biofiber has an important effect on the storage modulus ( $\left.E^{\prime}\right)$ as it increases considerably. According to the mechanical properties, Yong'modulus, tensile strength, strain at break and tenacity results supported that keratin biofiber acts as filler in TPS. On the other hand, when keratin fiber is added without FR in TPS, strain at break and tenacity are increased considerably. This is attributed to a good adhesion of the keratin biofiber with the polymer matrix.
\end{abstract}

Keywords: biofiber, keratin, biopolymer, thermal stability, mechanical reinforcement, tannery industry waste. 


\section{INTRODUCCIÓN}

Las fibras naturales en materiales compuestos han mostrado ser una alternativa muy atractiva a las fibras sintéticas convencionales como la fibra de vidrio o la de carbono. El uso potencial de los desechos agrícolas como refuerzos ecológicos y económicos en los materiales poliméricos ha establecido un beneficio en la explotación de los desechos agrícolas (Bilba et al., 2007; Faruk et al., 2012; Gurunathan et al., 2015). Las fibras naturales o biofibras de origen animal, que se generan de los procesos industriales, pueden ser una fuente muy importante de agentes de refuerzo, por ejemplo, el pelo de res que presenta un carácter hidrófobo por el alto contenido de queratina $(>90 \%)$, contrario a la celulosa, que tiene carácter hidrófilo; lo que representa una gran ventaja como agente de refuerzo en diversos polímeros. La queratina tiene una estructura bien ordenada, lo cual contribuye en la resistencia a la tensión, y posee alta relación de aspecto (longitud/diámetro) lo que le permite transferencia de carga efectiva de la matriz a la fibra. Se han encontrado importantes mejoras en las propiedades mecánicas de materiales compuestos a base de polímeros termoplásticos, incremento en la temperatura de transición vítrea y mayor estabilidad térmica evaluada por análisis termogravimétrico. Las principales aplicaciones que se proponen a los materiales compuestos a base de biofibra de queratina son en materiales de construcción, automotriz, materiales aislantes para usos eléctricos y acústicos, artículos desechables como platos, contenedores, membranas, filtros y aplicaciones biomédicas (Nanocoatings Inc., 2007). Por otro lado, la comercialización de los biopolímeros está limitada por la gran competencia del bajo costo de los polímeros "commodities" y de las excelentes propiedades de los polímeros de ingeniería, por lo que las investigaciones más recientes en el campo de los biopolímeros se han enfocado en el uso de polímeros biodegradables de bajo costo y en el refuerzo de propiedades mecánicas (Battegazzore et al., 2014). Los estudios sobre el comportamiento ante el fuego de los biopolímeros son escasos; sin embargo se han encontrado importantes resultados de materiales compuestos "eco-amigables" a base biopolímeros y fibra de coco (Gallo et al., 2013). Este tipo de materiales compuestos (biopolímero y fibra natural) preserva el carácter ecológico de los biopolímeros. Por otro lado, contribuye a la reducción de la descarga de contaminantes en el medio ambiente al utilizar fibras naturales a partir de desechos agrícolas.

El proceso del curtido del cuero genera una gran cantidad de desechos que representan un serio problema para el medio ambiente, por ejemplo, una tenería que procesa 1500 cueros diarios genera residuos de pelo de res húmedo de entre 4-5 toneladas diarias aproximadamente. El pelo es un residuo de desecho que contamina del medio ambiente, ya que se tiene que enviar a los rellenos sanitarios. Sin embargo, el pelo de la res es una fuente muy importante de biofibra de queratina. Una de las estrategias para reducir la carga de contaminantes es dar un valor agregado a los residuos de desechos. En el presente trabajo se evaluó el efecto de la biofibra de queratina en las propiedades térmicas, reológicas, dinámico-mecánicas y mecánicas de materiales compuestos. Estos materiales se obtuvieron a base de un biopolímero termoplástico de almidón en combinación con aditivos retardantes a la flama, libres de compuestos halogenados. El objetivo de la investigación es evaluar el posible uso de biofibra de queratina obtenida de los desechos de la industria de la curtiduría de México, como agente de refuerzo o carga, para dar valor agregado a estos por medio de la obtención de materiales sustentables.

\section{Parte experimental}

\section{MAteriales}

- Polímero termoplástico a base de almidón de maíz (TPS), Mater-Bi EF05S de la compañía NOVAMONT S.p.A., empleado como matriz biopolimérica.

- Biofibra de queratina, obtenida de los desechos de la industria de la curtiduría de México.

- Los aditivos retardantes a la flama libres de compuestos halogenados utilizados son: borato de melamina (MB), con $20 \%$ aproximadamente de contenido de $\mathrm{B}_{2} \mathrm{O}_{3}$, de la compañía Budenheim. Tri-hidróxido de aluminio (ATH) grado industrial con una pureza de $99.5 \%$. Borato de zinc (ZB), con contenido de $\mathrm{ZnO}$ de $37 \%$ y $47 \%$ de $\mathrm{B}_{2} \mathrm{O}_{3}$. Todos los aditivos se emplearon tal como se recibieron, sin ningún tratamiento posterior.

\section{MÉTOdOS DE OBTENCIÓN}

\section{Tratamiento DE LA BIOFibRA DE QUERATINA}

La biofibra de queratina (pelo de res) se obtuvo a partir de los desechos de la etapa de ribera, durante el proceso de curtido del cuero de una empresa curtidora de la región de León y Purísima del Rincón del Estado de Guanajuato, México. El proceso de curtido al cromo consiste principalmente en las siguientes etapas (Schubert, 1978; Wachsmann, 1999): 
a) Ribera. En esta etapa se llevan a cabo los procesos de remojo, pelambre, encalado, desencalado, rendido y desengrasado. El proceso de remojo sirve para rehidratar la piel que se ha conservado por salado y eliminar las proteínas solubles y otras impurezas. El pelambre se realiza para eliminar la queratina, principal componente del pelo y la epidermis. Durante el proceso de encalado se busca degradar la elastina y reticulina, saponificar las grasas, ablandar la estructura del colágeno y generar puntos reactivos. Esta etapa de ribera se lleva a cabo en un medio básico, en diferentes intervalos de $\mathrm{pH}(8,11-13$ y 8-9). Los productos que se emplean comúnmente para controlar el pH son: sulfuro y sulfhidrato de sodio, hidróxido de calcio, sulfato de amonio, bisulfito de sodio, enzimas proteolíticas y tensoactivos. Por la naturaleza de la etapa de ribera se generan diversos desechos con alta carga orgánica; uno de ellos son lodos que contiene el pelo de la res (biofibra de queratina) del proceso del pelambre.

b) Curtido. En esta etapa se difunde la sal de cromo hasta llegar a la molécula de colágeno. La etapa de curtido se lleva a cabo en un medio ácido $(\mathrm{pH} 3.0-$ 4.0). Los principales productos que se emplean para realizar el curtido son: sal común, ácido fórmico y sulfúrico, sulfato básico de cromo como curtiente y bicarbonato o formiato de sodio como basificantes.

c) Acabado en húmedo. Se llevan a cabo los procesos de neutralizado, recurtido, teñido, engrasado y fijado, para dar las propiedades de uso del cuero. Estos incluyen al compacto o esponjado, plano o relleno, firme o elástico, y finalmente el color.

d) Acabado en seco. Da apariencia y protección, dependiendo del uso que se le va a dar al cuero. El acabado se adecua según el uso común, como calzado, vestimenta, marroquinería o tapicería.

A los lodos obtenidos del proceso de pelambre (etapa de ribera), se les realizó un proceso de lavado para eliminar el hidróxido de calcio y reducir el contenido de las grasas. Posteriormente, la biofibra se secó al aire libre durante dos días y finalmente en una estufa a $40^{\circ} \mathrm{C}$ durante 12 h. Se determinó el contenido de nitrógeno total de la biofibra de queratina, ya que este es su principal componente. La determinación se realizó por medio del método Kjeldahl, de acuerdo con la norma NMX-AA-026-SCFI-2010, dando como resultado 91.0\% de nitrógeno total expresado como porciento de proteína. La biofibra de queratina utilizada tiene un tamaño muy variado, el diámetro va desde 16 hasta 80 micras y la longitud de 1-5 $\mathrm{mm}$ aproximadamente.

\section{EXTRUSIÓN REACTIVA}

Los materiales compuestos se obtuvieron mediante el proceso de extrusión reactiva, utilizando un extrusor doble husillo, Leistritz Micro 27, con una relación de longitud/diámetro (L/D) de 32, un diámetro de $27 \mathrm{~mm}$ y con 8 zonas de calentamiento. Los materiales se obtuvieron utilizando el siguiente perfil de temperatura: 17 0/180/185/185/195/195/190/185 ${ }^{\circ} \mathrm{C}$, con una velocidad de husillo de 120 revoluciones por minuto. El biopolímero TPS se secó previamente al proceso de extrusión. El secado del TPS se llevó a cabo en un secador por vacío, Maguire LPD-30, a una presión de vacío $0.5516 \mathrm{MPa}$ y a una temperatura de $105^{\circ} \mathrm{C}$ durante 15 min. El TPS, la biofibra de queratina y los aditivos retardantes a la flama se adicionaron conjuntamente en la zona de alimentación del extrusor. La concentración de la biofibra de queratina y de los aditivos retardantes a la flama utilizada se expresa en partes por cien de resina (phr). La composición de los materiales obtenidos se describe en las tablas 1 y 2 .

\section{MOLDEO POR INYECCIÓN}

Las muestras para la evaluación de propiedades mecánicas y dinámico-mecánicas se obtuvieron por el proceso de moldeo por inyección. Los especímenes para evaluar las propiedades mecánicas se obtuvieron de acuerdo con la norma ASTM D638. El proceso de moldeo por inyección se llevó a cabo utilizando una inyectora Milacron TM55, con el siguiente perfil de temperatura: 175/178/ $180 / 185^{\circ} \mathrm{C}$, a una velocidad y presión de inyección de 95 $\mathrm{mm} / \mathrm{s}$ y $10 \mathrm{MPa}$, respectivamente.

\section{Métodos de CARACTERIZACIÓN}

Las propiedades mecánicas de tensión se evaluaron en una máquina Instron modelo 5565 siguiendo el procedimiento establecido en la norma ASTM D638-10 "Standard Test Method for Tensile Properties of Plastics", con una rapidez de deformación de $50 \mathrm{~mm} / \mathrm{min}$ (velocidad de la cruceta) y utilizando probetas de dimensiones de acuerdo con el tipo I (sección 6 de la norma). Los análisis termogravimétricos se realizaron en un equipo TA-Instrument 2950, con una velocidad de calentamiento de $10^{\circ} \mathrm{C} / \mathrm{min}$ en atmósfera de nitrógeno, en un intervalo de temperatura de $30-600^{\circ} \mathrm{C}$, el tamaño de la muestra fue de 10-14 mg. Las mediciones reológicas se llevaron a cabo en un reómetro de esfuerzo controlado TA-Instrument G2 utilizando geometría de platos paralelos de $25 \mathrm{~mm}$ de diámetro, una distancia entre platos de $0.75 \mathrm{~mm}$ y una cámara con sistema de horno de ca- 
lentamiento (TA Extended temperature system). Las muestras se llevaron a una temperatura inicial de $200^{\circ} \mathrm{C}$ durante dos minutos con la intención de que todas las muestras tuvieran la misma historia térmica previa al barrido de temperatura. Las mediciones se realizaron a una velocidad de calentamiento de $10^{\circ} \mathrm{C} / \mathrm{min}$ y con una frecuencia angular de $1 \mathrm{rad} / \mathrm{s}$, a una deformación de $3 \%$ que se estableció previamente dentro del intervalo viscoelástico lineal. Las pruebas dinámico-mecánicas se realizaron utilizando un analizador dinámico-mecánico TA-Instrument Q800 a una frecuencia $(1.0 \mathrm{~Hz})$, con una amplitud de $10 \mu \mathrm{m}$ y con una velocidad de calentamiento de $3^{\circ} \mathrm{C} / \mathrm{min}$. Las muestras fueron rectangulares con dimensiones de $13 \mathrm{~mm}$ de ancho, $60 \mathrm{~mm}$ de largo y un espesor de $3 \mathrm{~mm}$, la geometría empleada fue la de un solo punto fijo (single cantiliver).

\section{RESULTADOS Y DISCUSIÓN}

\section{TPS CON BIOFIBRA DE QUERATINA Y BORATO DE MELAMINA}

En la figura 1 se presenta la rapidez de pérdida de masa respecto a la temperatura de la biofibra de queratina y el Borato de Melamina (MB). Se puede observar que la biofibra de queratina presenta mayor estabilidad térmi- ca de acuerdo con el MB. La temperatura de degradación máxima $\left(\mathrm{T}_{\max }\right)$, donde ocurre la principal pérdida de masa, de la biofibra es ligeramente mayor $\left(304^{\circ} \mathrm{C}\right)$ respecto al $\mathrm{MB}$, que presentó una $\mathrm{T}_{\max }$ de $301^{\circ} \mathrm{C}$. En la tabla 3 se presenta la $T_{\max }$ rapidez de pérdida de masa y la formación de residuos a $600^{\circ} \mathrm{C}$, obtenido del análisis termogravimétrico de la biofibra de queratina, $\mathrm{MB}, \mathrm{y}$ los materiales compuestos a base de TPS. En cuanto a la rapidez de pérdida de masa (en $\mathrm{T}_{\max }$ ), esta es claramente menor en el caso de la biofibra de queratina $(0.584$ $\left.\% /{ }^{\circ} \mathrm{C}\right)$ de acuerdo con el $\mathrm{MB}\left(1.386 \% /{ }^{\circ} \mathrm{C}\right)$. La biofibra de queratina presenta mayor formación de residuos $(21.6 \%)$ respecto al MB (19.1\%). Los resultados obtenidos muestran que la biofibra de queratina presenta mayor estabilidad térmica que el MB. Este comportamiento se atribuye a la composición de la queratina, ya que al tratarse de una proteína, los principales grupos de los aminoácidos contribuyen en la formación de compuestos fenoles y aromáticos. Estos compuestos son estructuras estables a alta temperatura, que se forman lentamente arriba de $300^{\circ} \mathrm{C}$ durante la degradación de la queratina (Brebu y Spiridon, 2011). Este comportamiento de la biofibra representa una ventaja para utilizarse como aditivo retardante a la flama (Wrześniewska et al., 2014). La degradación principal

Tabla 1. Descripción de materiales a base de TPS, biofibra de queratina y MB

\begin{tabular}{ccc}
\hline $\begin{array}{c}\text { Identificación de la } \\
\text { muestra }\end{array}$ & $\begin{array}{c}\text { Biofibra de } \\
\text { queratina } \\
\text { (phr) }\end{array}$ & $\begin{array}{c}\text { MB } \\
\text { (phr) }\end{array}$ \\
\hline TPS-A1 & 3 & - \\
TPS-A2 & 5 & - \\
TPS-A3 & - & 3 \\
TPS-A4 & - & 5 \\
TPS-A5 & - & 10 \\
TPS-A6 & 3 & 5 \\
TPS-A7 & 5 & 3 \\
TPS-A8 & 5 & 5 \\
\hline
\end{tabular}

\begin{tabular}{cccc}
$\begin{array}{c}\text { Tabla 2. Descripción de materiales a base de TPS, biofibra de queratina, ATH y ZB } \\
\text { muestra }\end{array}$ & $\begin{array}{c}\text { Biofibra de } \\
\text { queratina (phr) }\end{array}$ & $\begin{array}{c}\text { ATH } \\
(\mathrm{phr})\end{array}$ & $\begin{array}{c}\text { ZB } \\
(\mathrm{phr})\end{array}$ \\
\hline TPS-B1 & - & 50 & 3 \\
TPS-B2 & 10 & - & - \\
TPS-B3 & 5 & - & - \\
TPS-B4 & 3 & - & - \\
TPS-B5 & 10 & 30 & 3 \\
TPS-B6 & 5 & 30 & 3 \\
TPS-B7 & 3 & 30 & 3 \\
\hline
\end{tabular}


del MB ocurre entre $220-300^{\circ} \mathrm{C}$, en la cual se atribuye a la dehidratación del ácido ortobórico. Posteriormente se forma el ácido metaborico $\left(\mathrm{HBO}_{2}\right)$ y finalmente se forma óxido de boro $\left(\mathrm{B}_{2} \mathrm{O}_{3}\right)$. Al mismo tiempo, más de la mitad de la melamina contenida en el MB se sublima parcialmente, la otra parte de la melamina se atribuye a la formación de grupos melem y melon en los residuos (Costa et al., 1990; Hoffendahl et al., 2014).

En la figura 2 se presenta la rapidez en la pérdida de masa respecto a la temperatura de los materiales a base de TPS con 3 y 5 phr de biofibra de queratina. Los resultados muestran que los materiales que contienen biofibra de queratina presentan ligeramente menor rapidez de descomposición, de acuerdo con el TPS, en especial con el incremento del contenido de biofibra (5 phr). En relación con la tabla 3 , los materiales compuestos con biofibra de queratina muestran una $\mathrm{T}_{\max }$ ligeramente menor que el TPS, $361^{\circ} \mathrm{C}$ el TPS-A1 y $360^{\circ} \mathrm{C}$ el TPS-A2, respectivamente a $363^{\circ} \mathrm{C}$ del TPS. Sin embargo, la formación de residuos a $600^{\circ} \mathrm{C}$ es mayor con la adición de la biofibra de queratina, $6.8 \%$ cuando se adiciona $3 \mathrm{phr}$ de biofibra y $9.5 \%$ cuando se adicionan $5 \mathrm{phr}$ de biofibra. Esto indica que la biofibra de queratina contribuye en la estabilidad térmica del TPS, lo cual se atribuye a la composición de la queratina.

En la figura 3 se presenta la rapidez de pérdida de masa en proporción a la temperatura de los materiales a base de TPS con 3, 5 y 10 phr de MB (TPS-A3, TPS-A4 y TPS-A5). Los resultados muestran que, los materiales que contienen MB presentan mayor rapidez de descomposición, respecto a la del TPS. Los resultados muestran que $\mathrm{T}_{\max }$ es menor para los tres casos, respectivamente a la $\mathrm{T}_{\max }$ del TPS $\left(363^{\circ} \mathrm{C}\right)$. En cuanto a la formación de residuos, los materiales con $\mathrm{MB}$ presentan un porcentaje proporcional a la concentración del aditivo. Los resultados indican que el aditivo MB no contri-

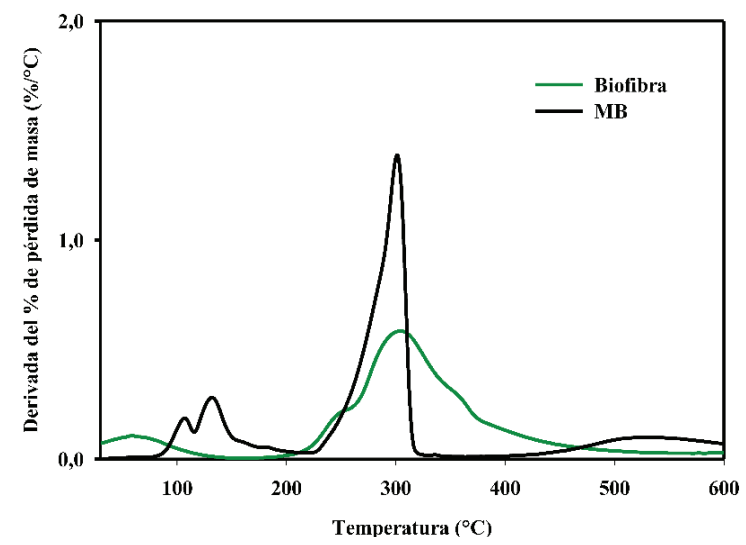

Figura 1. Rapidez en la pérdida de masa respecto a la temperatura de la biofibra de queratina y $\mathrm{MB}$ buye de forma importante en la estabilidad térmica de los materiales compuestos a base de TPS, lo cual se atribuye a la alta rapidez de pérdida de masa que presenta en la temperatura de degradación principal.

De acuerdo con la figura 4 , los materiales que contienen 5 phr de biofibra de queratina, con 3 y 5 phr de $\mathrm{MB}$, muestran mayor rapidez de descomposición respecto al TPS. Cuando la concentración de biofibra de queratina es menor ( $3 \mathrm{phr}$ ), en combinación con $5 \mathrm{phr}$ de MB (TPS-A6), la rapidez de pérdida de masa disminuye de 1.38 a $1.16 \% /{ }^{\circ} \mathrm{C}$, de acuerdo con el TPS. Sobre la tabla 3, la formación de residuos es notoriamente mayor cuando la biofibra de queratina se encuentra en baja concentración ( $3 \mathrm{phr}$ ) y en combinación con 5 phr de MB. La formación de residuos para el TPS-A6, A7 y A8 es de 31.0, 9.8 y $13.8 \%$, respectivamente. Las $\mathrm{T}_{\max }$ para estos materiales (TPS-A6, A7 y A8) son de 355, 357 y $355^{\circ} \mathrm{C}$, respectivamente. De acuerdo con los resultados de rapidez de pérdida de masa y formación de residuos a $600^{\circ} \mathrm{C}$, la combinación de una mínima concentración de biofibra de queratina ( $3 \mathrm{phr}$ ) con 5 phr de MB, proporciona mayor estabilidad térmica a los materiales compuestos a base de TPS; lo cual se atribuye a que a bajas concentraciones la biofibra de queratina y el MB promueven la formación de estructuras entrecruzadas durante el proceso de degradación térmica, debido al alto contenido de nitrógeno de ambos componentes (Shen et al., 2010).

Tabla 3. Temperatura de degradación máxima $\left(\mathrm{T}_{\max }\right)$, rapidez en la pérdida de masa y formación de residuos a $600^{\circ} \mathrm{C}$ de la biofibra de queratina, MB y los materiales compuestos a base de TPS

\begin{tabular}{cccc}
\hline $\begin{array}{c}\text { Identificación de la } \\
\text { muestra }\end{array}$ & $\begin{array}{c}\mathrm{T}_{\max } \\
\left({ }^{\circ} \mathrm{C}\right)\end{array}$ & $\begin{array}{c}\text { Rapidez en } \\
\text { la pérdida de } \\
\text { masa en } \mathrm{T}_{\max } \\
\left(\% /{ }^{\circ} \mathrm{C}\right)\end{array}$ & $\begin{array}{c}\text { Residuos } \\
\mathrm{a} 600^{\circ} \mathrm{C} \\
(\%)\end{array}$ \\
\hline $\begin{array}{c}\text { Biofibra de } \\
\text { queratina }\end{array}$ & 304 & 0.584 & 21.6 \\
MB & 301 & 1.386 & 19.1 \\
TPS & 363 & 1.385 & 4.5 \\
TPS-A1 & 361 & 1.302 & 6.8 \\
TPS-A2 & 360 & 1.178 & 9.5 \\
TPS-A3 & 356 & 1.522 & 7.7 \\
TPS-A4 & 353 & 1.648 & 10.2 \\
TPS-A5 & 351 & 1.577 & 12.7 \\
TPS-A6 & 355 & 1.161 & 31.0 \\
TPS-A7 & 357 & 1.459 & 9.8 \\
TPS-A8 & 355 & 1.516 & 13.8 \\
\hline
\end{tabular}




\section{TPS CON BIOFIBRA DE QUERATINA, HIDRÓXIDO DE ALUMINIO Y BORATO DE ZINC}

En la figura 5 se presenta la viscosidad compleja respecto a la temperatura de los materiales a base de TPS, biofibra de queratina, ATH y ZB. El incremento súbito de la viscosidad compleja indica la temperatura de degradación principal, en donde se inicia la formación de estructuras complejas que son resistentes a la deformación y a la degradación térmica. Generalmente, en los polímeros termoplásticos se presenta la degradación de las cadenas poliméricas con el aumento de temperatura y con ello incrementa la fluidez (baja la viscosidad). Por lo tanto, la evaluación de la viscosidad compleja en consideración con la temperatura es una forma de estudiar la estabilidad térmica de los materiales poliméricos (Marosföi et al., 2006). En la figura 5 se puede observar que el TPS muestra una disminución de la viscosidad compleja con el incremento de la temperatura, particularmente a partir de $250^{\circ} \mathrm{C}$ hasta $325^{\circ} \mathrm{C}$, temperatura en la cual la viscosidad compleja presenta un incremento súbito, lo cual indica la formación de estructuras resistentes a la temperatura. Este comportamiento es característico de polímeros, como es el caso de las poliolefinas, polímeros estirénicos y vinílicos, los cuales son altamente inflamables al contacto con el fuego (Price et al., 2001). La adición de biofibra de queratina en el TPS, muestra una influencia importante en la viscosidad compleja en relación con la temperatura, particularmente con 10 y 3 phr. El material TPS-B4, presenta un incremento súbito de la viscosidad compleja a $350^{\circ} \mathrm{C}$. A pesar de que dicho incremento ocurre a una temperatura mayor, de acuerdo con el TPS, la viscosidad compleja permanece menor en todo el intervalo de temperatura

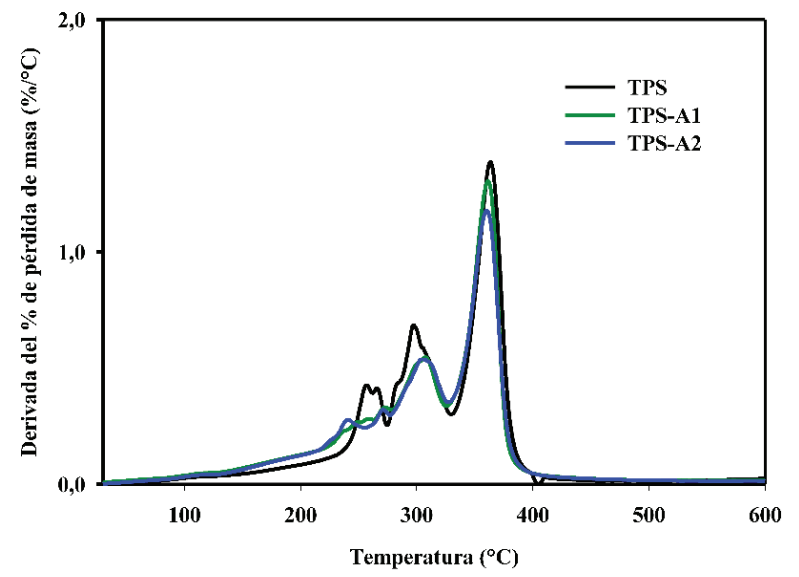

Figura 2. Rapidez en la pérdida de masa de acuerdo con la temperatura de los materiales a base de TPS con 3 y 5 phr de biofibra de queratina de prueba. El material TPS-B3, muestra un comportamiento muy similar al TPS; el incremento de la viscosidad compleja se presenta a partir de $325^{\circ} \mathrm{C}$. Los resultados de la evaluación de la viscosidad compleja de los materiales TPS-B3 y TPS-B4, muestran que a 3 y $5 \mathrm{phr}$ de biofibra de queratina, no existe formación de estructuras que impacten en la viscosidad a alta temperatura. Sin embargo, cuando se adiciona biofibra en mayor concentración (10 phr), se puede observar que el TPS-B2, muestra un importante aumento en la viscosidad compleja a una temperatura de aproximadamente $310^{\circ} \mathrm{C}$, lo que indica que a esta temperatura se inicia la formación de estructuras complejas y estables. Este resultado muestra que la adición de 10 phr de biofibra proporciona mayor estabilidad térmica al TPS. Este mismo comportamiento se presentó con los materiales que contienen ATH ( $30 \mathrm{phr})$ y ZB ( 3 phr) en combinación con 5 y 3 phr de biofibra (TPS-B6 y TPS-B7) en donde la viscosidad compleja aumenta a la misma temperatura $\left(310^{\circ} \mathrm{C}\right)$. Por lo tanto, la biofibra de queratina adicionada en $10 \mathrm{phr}$, muestra el mismo efecto en la estabilidad térmica que los aditivos retardantes a la flama (ATH y ZB). Estos resultados abren la posibilidad de que los desechos de la industria de la curtiduría tengan una aplicación como aditivos retardantes a la flama sustentables.

La figura 6 muestra el módulo de almacenamiento $\left(E^{\prime}\right)$ en comparación con la temperatura de los materiales compuestos a base de TPS, biofibra de queratina, ATH y ZB. De forma general se puede observar que la adición de los aditivos retardantes a la flama y de la biofibra de queratina en diferentes concentraciones tienen un efecto importante en el aumento del $E^{\prime}$, lo cual indica la obtención de un material con mayor rigidez

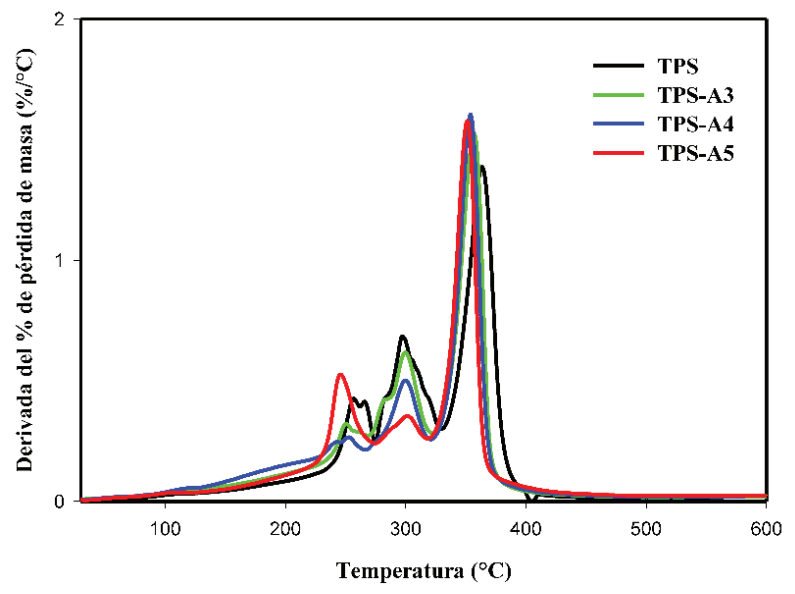

Figura 3. Rapidez en la pérdida de masa respecto a la temperatura de los materiales a base de TPS con diferentes concentraciones de MB 
respecto al TPS. En todos los casos de los materiales compuestos, la transición de la zona vítrea a la zona viscoelástica, no es tan pronunciada como en el caso de materiales cristalinos o semi-cristalinos. En el caso de los materiales a base de TPS, estos presentan una transición de la zona vítrea a la zona viscoelástica amplia, que inicia a muy baja temperatura $\left(35^{\circ} \mathrm{C}\right)$ y finaliza en $75^{\circ} \mathrm{C}$ aproximadamente. Como se puede observar en la figura 6, el alto contenido ATH (50 phr), produce un incremento considerable del $\mathrm{E}^{\prime}$, respecto al TPS, de 300 hasta $700 \mathrm{MPa}$ (aproximadamente un incremento de $133 \%$ ), que es característico de los materiales altamente cargados. De acuerdo con el efecto de la concentración de la biofibra de queratina, se observa que no tiene un importante efecto en el aumento del $E^{\prime}$, ya que independientemente del contenido de biofibra el aumento de $\mathrm{E}^{\prime}$ es similar. Los materiales TPS-B2, TPS-B3 y TPS-B4 presentan un $\mathrm{E}^{\prime}$ de $400 \mathrm{MPa}$ aproximadamente (un incremento de 33\%), lo cual significa que con una mínima concentración de biofibra ( 3 phr) se logra obtener un material con mayor rigidez que el TPS virgen. Este comportamiento se atribuye a que la biofibra de queratina tiene amplia relación de aspecto, ya que la biofibra tiene un diámetro de 16-80 micras y una longitud de 1-5 mm aproximadamente (Nanocoatings Inc., 2007). La combinación de la biofibra de queratina en diferentes concentraciones (10, 5 y 3 phr) con $30 \mathrm{phr}$ de ATH y $3 \mathrm{phr}$ de ZB, induce un aumento en el $\mathrm{E}^{\prime}$ muy similar en los tres casos, alrededor de $600 \mathrm{MPa}$. Estos resultados muestran que el aumento del E' es proporcional al contenido del aditivo retardante a la flama e independiente de la concentración de la biofibra de queratina, lo cual muestra que el ATH y el ZB actúan como cargas que inducen un mayor efecto que la biofibra.

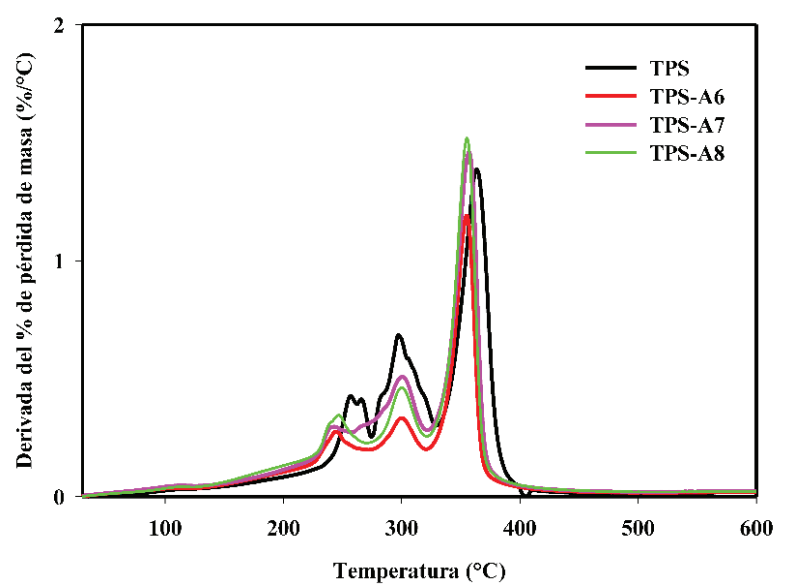

Figura 4. Rapidez en la pérdida de masa de acuerdo con la temperatura de los materiales a base de TPS con diferentes concentraciones de biofibra de queratina y MB
En la tabla 4 se presentan los resultados de las propiedades mecánicas de los materiales a base de TPS, biofibra de queratina, ATH y ZB. Como se puede observar, la composición y concentración de los materiales tienen un efecto muy importante en las propiedades mecánicas del TPS. La alta concentración del ATH (50 phr) provoca una reducción muy importante en la resistencia a la tensión, deformación a la ruptura y en la tenacidad, de acuerdo con el TPS virgen. La resistencia a la tensión disminuye de 23 a $12 \mathrm{MPa}$, la deformación a la ruptura se reduce de $>791$ hasta $24 \%$; sin embargo el módulo de Young se incrementa de 89 a 202 MPa. Estas propiedades son características de los materiales rígidos y frágiles, alto módulo elástico y baja deformación, lo que se atribuye a la alta carga del ATH. De acuerdo con el efecto de la concentración de la biofibra de queratina en las propiedades mecánicas, se puede observar que el módulo de Young aumenta ligeramente, respecto al TPS virgen, con la adición de biofibra de queratina. Dicho aumento es proporcional a la concentración de biofibra de queratina; ya que de 89 MPa del TPS aumenta a 95, 100 y 107 MPa con 3, 5 y 10 phr de biofibra, respectivamente (TPS-B4, TPS-B3 y TPS-B2). El efecto de la biofibra de queratina en la deformación a la ruptura y la tenacidad, muestra que se obtienen materiales dúctiles que los material con alta concentración de aditivo retardante a la flama; ya que presentan una deformación a la ruptura de 349, 448 y $373 \%$ y una tenacidad de 44,59 y $14 \mathrm{MPa}$, con 3, 5 y 10 phr de biofibra, respectivamente. La mayor deformación a la ruptura y tenacidad que se presentó es cuando contiene $5 \mathrm{phr}$ de biofibra (TPS-B3). Estas propiedades se atribuyen a que la biofibra de queratina logra una buena adhesión con la matriz polimérica (Barone, 2005).

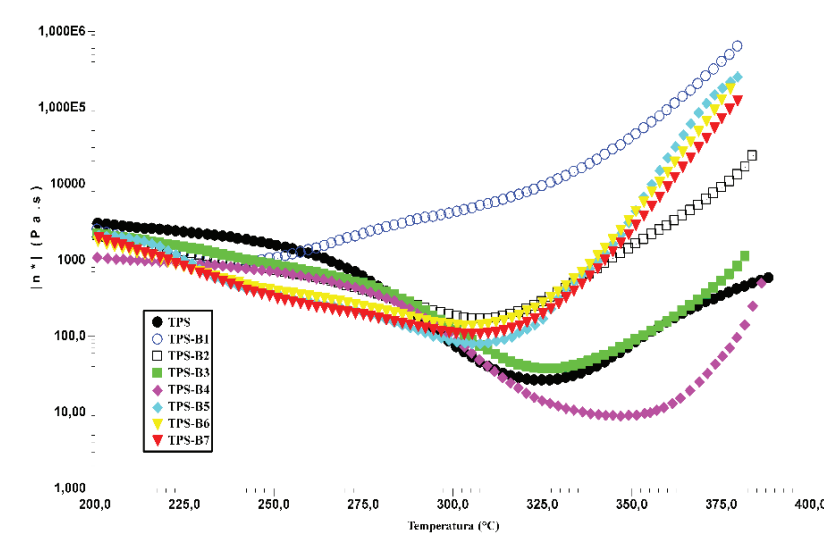

Figura 5. Viscosidad compleja respecto a la temperatura de los materiales a base de TPS, biofibra de queratina, ATH y ZB 


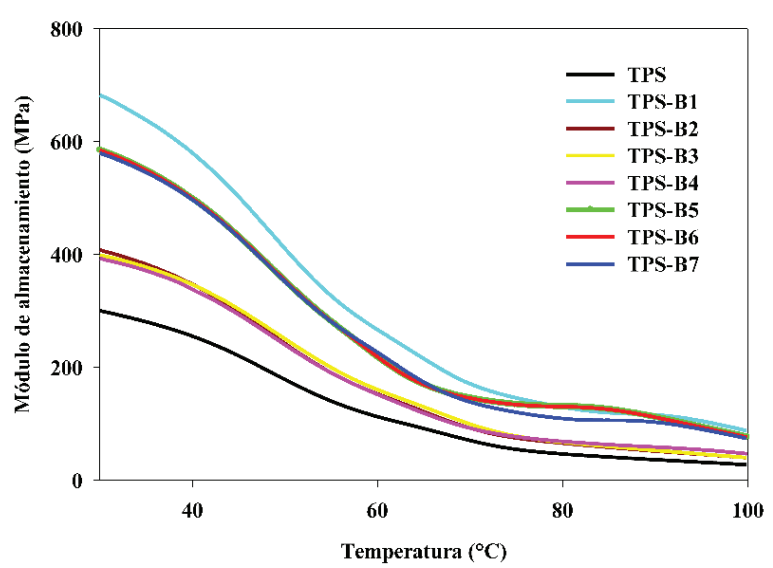

Figura 6. Módulo de almacenamiento respecto a la temperatura de los materiales a base de TPS, biofibra de queratina, ATH y ZB

Tabla 4. Propiedades mecánicas de los materiales a base de TPS, biofibra de queratina, ATH y ZB

\begin{tabular}{ccccc}
\hline $\begin{array}{c}\text { Identificación de } \\
\text { la muestra }\end{array}$ & $\begin{array}{c}\text { Módulo de Young } \\
\text { (MPa) }\end{array}$ & $\begin{array}{c}\text { Resistencia a la tensión } \\
\text { en la carga máxima } \\
(\mathrm{MPa})\end{array}$ & $\begin{array}{c}\text { Deformación a la } \\
\text { ruptura } \\
(\%)\end{array}$ & Tenacidad (MPa) \\
\hline TPS & 89 & 23 & $>791$ & $>133$ \\
TPS-B1 & 203 & 12 & 24 & 2 \\
TPS-B2 & 107 & 14 & 373 & 14 \\
TPS-B3 & 100 & 16 & 448 & 59 \\
TPS-B4 & 95 & 16 & 349 & 44 \\
TPS-B5 & 192 & 12 & 85 & 9 \\
TPS-B6 & 179 & 12 & 57 & 6 \\
TPS-B7 & 183 & 13 & 88 & 11 \\
\hline
\end{tabular}

La combinación de biofibra de queratina y los retardantes a la flama ATH y ZB, produce materiales rígidos y frágiles (ya que se incrementa el módulo de Young, proporcionalmente de acuerdo con el contenido de biofibra) y disminuyendo la resistencia a la tensión, la deformación a la ruptura y la tenacidad, independientemente del contenido de biofibra. La resistencia a la tensión disminuye prácticamente a la mitad con la adición del ATH y el ZB, respecto al TPS, lo cual implica que los materiales tienen una menor capacidad de absorción de esfuerzos. Este comportamiento se atribuye a la presencia del ATH en alta concentración (30 phr).

\section{Conclusiones}

En el presente trabajo se evaluó el efecto de la biofibra de queratina en la producción de nuevos materiales. Esta se obtuvo de los desechos de la curtiduría en combinación con aditivos retardantes a la flama libres de compuestos halogenados. Los materiales compuestos a base de TPS, biofibra y MB se caracterizaron por análisis termogravimétrico y los materiales a base de TPS, biofibra, ATH y ZB se caracterizaron por medio de pruebas dinámico-mecánicas, mecánicas y reológicas de materiales compuestos a base de TPS. Del análisis termogravimétrico, se encontró que la biofibra de queratina proporciona mayor estabilidad térmica en el TPS, respecto al aditivo comercial borato de melamina $(\mathrm{MB})$, ya que disminuye la rapidez de pérdida de masa y promueve la formación de residuos del TPS. La evaluación de la viscosidad compleja de acuerdo con la temperatura demostró que la adición de 10 phr de biofibra de queratina promueve la formación de estructuras complejas resistentes a la temperatura, lo cual es un comportamiento similar al de los materiales que contienen aditivos retardantes a la flama comerciales. El análisis dinámico-mecánico mostró que la adición de biofibra de queratina en el TPS incrementa el módulo 
de almacenamiento ( $\left.E^{\prime}\right)$, respecto al TPS virgen, lo que muestra que la biofibra de queratina actúa como carga. En cuanto a las propiedades mecánicas, los resultados mostraron que la biofibra de queratina adicionada en bajas concentraciones ( 3 y 5 phr), promueve la obtención de materiales rígidos, pero con mejor deformación, ya que la deformación a la ruptura y la tenacidad incrementan de acuerdo con los materiales altamente cargados con aditivos retardantes a la flama. Estos resultados indican que la biofibra de queratina presenta cierta compatibilidad con la matriz polimérica, debido posiblemente al carácter hidrófobo de la biofibra. El estudio realizado en el presente trabajo señala que la biofibra de queratina, obtenida de los desechos de la industria de la curtiduría, podría utilizarse como aditivo sustentable en materiales a base de biopolímeros.

\section{AgradeCimientos}

Al Consejo Nacional de Ciencia y Tecnológica del Estado de Guanajuato (CONCYTEG), a través del proyecto Convenio número 090/2015CIATEC, por otorgar los recursos para la realización del trabajo de investigación. A la M. en A. Damaris Cabrero-Palomino por la realización de los análisis termogravimétricos.

\section{Nomenclatura}

TPS Biopolímero termoplástico a base de almidón

FR Aditivo retardante a la flama

ATH Tri-hidróxido de aluminio

MB Borato de melamina

$\mathrm{T}_{\max } \quad$ Temperatura de degradación máxima determinada por análisis termogravimétrico

phr Partes por cien de resina

\section{RefERENCIAS}

Barone J.R. Polyethylene/keratin fiber composites with varying polyethylene crystallinity. Composites, Part A, volumen 36, 2005: 1518-1524.

Battegazzore D., Alongi J., Frache A. Poly(lactic acid)-Based Composites Containing Natural Fillers: Thermal, Mechanical and Barrier Properties. Journal of Polymers and the Environment, volumen 22, 2014: 88-98.

Bilba K., Arsene M. A., Ouensanga A. Study of banana and coconut Wbers Botanical composition, thermal degradation and textural observations. Bioresource Technology, volumen 98, 2007: 58-68.
Brebu M. y Spiridon I. Thermal degradation of keratin waste. Journal of Analytical and Applied. Pyrolysis, volumen 91, 2011: 288295.

Costa L., Camino G., Luda di Cortemiglia M.P. Mechanism of termal degradtion of fire-retardant melamine salts, en: Gordon N.L. Fire and polymer hazards identification and prevention, Whashington, DC, American Chemical Society, 1990. pp. 231237.

Faruka O., Bledzkia A.K., Fink H-A., Sain M. Biocomposites reinforced with natural fibers: 2000-2010. Progress in Polymer Science, volumen 37, 2012: 1552-1596.

Gallo E., Sanchez-Olivares G., Schartel B. Flame retardancy of starch-based biocomposites-aluminum hydroxide-coconut fiber synergy. Polimery, volumen 58 (número 5), 2013: 395-402.

Gurunathan T., Mohanty, Nayak S.K. A review of the recent developments in biocomposites based on natural fibres and their application perspectives. Composites, Part A, volumen 77, 2015: 1-25.

Hoffendahl C., Duquesne S., Fontaine G., Bourbigot. Decomposition mechanism of melamine borate in pyrolytic and thermooxidative conditions. Thermochimica Acta, volumen 590, 2014: 73-83.

Marosföi B., Matko Sz., Anna P., Marosi G. Fire retarded polymer nanocomposites. Current Applied Physics, volumen 6, 2006: 259-261.

Polymer reinforcement, Revista de CG2 Nanocoatings Inc [en línea] 2007 [fecha de consulta: mayo de 2012]. Disponible en: http:// www.cg2nanocoatings.com/CG2PolymerReinforcement

Price D., Anthony G., Carty P. Introduction: polymer combustion, condensed phase pyrolysis and smoke formation, en: Horrocks A.R., Price D. Fire retardant materials, Cambridge, Woodhead Publishing Limited and CRC Press LLC, 2001. pp. 3-13.

Shen K.K., Kochesfahani S.H., Jouffret F. Boron-Base Flame retardants and Flame Retardancy, en: Wilkie C.A, Morgan A.B. Fire Retardancy of Polymeric Materials, Boca Raton, CRC Press, 2010. pp. 223-225.

Schubert A. The manufacture of leather from calfskin, en: O'Flaherty F., Roddy W.T., Lollar R.M. (Eds.), The chemistry and technology of leather (Vol. 3). Robert E. Krieger Publishing company, Malabar, 1978, pp. 178-234.

Wachsmann H.M. En: Leafe M.K. (Ed.), Leather Technologist pocket book. Society of Leather Technologists, Withernsea, UK, 1999. pp. 85-98.

Wrześniewska-Tosik K., Zajchowski S., Bryśkiewicz A., Ryszkowska J. Feathers as a flame-retardant in elastic polyurethane foam. FIBRES \& TEXTILES in Eastern Europe, volumen 22, 2014: 119-128. 


\section{Citación sugerida:}

\section{Citación estilo Chicago}

Sánchez-Olivares, Guadalupe, Luis Medina-Torres, Fausto Calderas, Antonio Sánchez-Solis, Rigel Leonardo Moreno-Morales, Víctor RamírezGonzález, Octavio Manero. Efecto de la biofibra de queratina en las propiedades de un biopolímero termoplástico: Estudio preliminar. Ingeniería Investigación y Tecnología, XVIII, 04 (2017): 369-378.

\section{Citación estilo ISO 690}

Sánchez-Olivares G., Medina-Torres L., Calderas F., Sánchez-Solis A., Moreno-Morales R.L., Ramírez-González V., Manero O. Efecto de la biofibra de queratina en las propiedades de un biopolímero termoplástico: Estudio preliminar. Ingeniería Investigación y Tecnología, volumen XVIII (número 4), octubre-diciembre 2017: 369-378.

\section{Semblanzas de los autores}

Guadalupe Sánchez-Olivares. Obtuvo el doctorado en ingeniería química por la UNAM. Estancia de investigación en el Politécnico di Torino, Alessandria, Italia. Posdoctorado en el Federal Institute for Materials Research and Testing, Berlín, Alemania. Es miembro del SNI nivel I, su principal línea de investigación es el comportamiento ante el fuego de polímeros, textiles y cuero.

Luis Medina-Torres. Miembro del SNI nivel I. Fue acreedor a la medalla al mérito universitario por mejor promedio en la UAMIztapalapa. Premio estatal de ciencia, tecnología e innovación del estado de Durango 2015. Su área de investigación se centra en reología de sistemas heterogéneos y mezclado.

Fausto Calderas. Doctor en ingeniería química por parte de UNAM. Es experto en el área de reología con diez años de experiencia en manejo de reómetros capilares, de esfuerzo controlado y de deformación controlada. Miembro del SNI nivel I.

Antonio Sánchez-Solis. Doctorado en ingeniería química por la UNAM. Cuenta con el posdoctorado en el Industrial Materials Institute, Quebéc. Canada. Es miembro del SNI Nivel I y asesor de la industria del plástico por más de 30 años. Es experto en el procesamiento de materiales poliméricos, compuestos y nanocompuestos.

Rigel Leonardo Moreno-Morales. Maestro en ciencia e ingeniería de materiales por el Instituto de Investigaciones en Materiales de la UNAM y candidato a doctor en ciencia e ingeniería de materiales por el Instituto de Investigaciones en Materiales de la UNAM, especialista en el comportamiento reológico de fluidos biológicos.

Víctor Ramírez-González. Asesor técnico en curtiduría, ha participado en diversos proyectos de investigación y desarrollo tecnológico para la industria de la curtiduría de la región de León, Guanajuato.

Octavio Manero. Doctor en matemáticas aplicadas por la Universidad de Gales, Gran Bretaña. Posdoctorado en el Instituto Tecnológico de California, USA, es miembro del SNI Nivel III. Su línea de investigación es en ciencia y tecnología de fluidos complejos. Obtuvo el Premio Nacional de Química 2002 y el Premio Nacional de Ciencias y Artes, 2003. 